\title{
Arbeitsintensität und ihre Determinanten. Eine Übersicht über das Analysepotential von Erwerbstätigenbefragungen
}

\author{
Lena Hünefeld ${ }^{1}$. Elke Ahlers ${ }^{2}$. Serife Erol Vogel ${ }^{2}$. Sophie-Charlotte Meyer ${ }^{1}$
}

Angenommen: 15. Januar 2021 / Online publiziert: 5. Oktober 2021

(c) Der/die Autor(en) 2021

\section{Zusammenfassung}

Eine langanhaltende hohe Arbeitsintensität kann eine gesundheitsgefährdende Belastung für Beschäftigte darstellen. Für eine angemessene Gestaltung der Arbeit sind Kenntnisse von Determinanten dauerhaft erhöhter Arbeitsintensität von zentraler Bedeutung. Dieser Beitrag beschäftigt sich mit der Frage, welche empirischen Grundlagen zur Verfügung stehen, um die Verbreitung hoher Arbeitsintensität in Deutschland zu beschreiben und insbesondere potenzielle Determinanten zu identifizieren. Hierfür wurden sechs Erwerbstätigenbefragungen systematisch analysiert. Es konnten insgesamt 662 Fragebogenitems zum Thema „Arbeitsintensität und ihre Determinanten“ identifiziert und anhand von 11 Hauptkategorien - sechs Kategorien für Arbeitsintensität und fünf für Determinanten - und mehr als 65 Unterkategorien klassifiziert werden. Trotz empirischer Lücken zeigt sich damit, dass in Deutschland eine breite Datenbasis zur Analyse des Themas Arbeitsintensität und deren Determinanten zur Verfügung steht. In zukünftigen Erhebungen sollten auch Informationen zur Leistungspolitik, wie Kundenorientierung oder auch Dienstreisen, Zielvereinbarungen, dem Maß der Ergebnisorientierung und auch Eigenverantwortung als mögliche Determinanten von Arbeitsintensität erhoben werden. Ebenfalls sollte die ausführliche Erhebung von betrieblichen Rahmenbedingungen, wie die Unternehmensform oder auch existierende Personalengpässe stärker mitbedacht werden.

Praktische Relevanz: Die vorliegende Studie verdeutlicht, welche Datenbasis in Deutschland vorhanden ist, um Determinanten von Arbeitsintensität zu beleuchten und somit auch Gestaltungspotentiale für eine angemessene Arbeitsintensität zu identifizieren.

Schlüsselwörter Arbeitsanforderung · Arbeitsbelastungen · Arbeitsgestaltung · Gesundheit · Erwerbstätigenbefragungen

Dr. Lena Hünefeld

Huenefeld.Lena@baua.bund.de

1 Bundesanstalt für Arbeitsschutz und Arbeitsmedizin (BAuA),

Friedrich-Henkel-Weg 1-25, 44149 Dortmund, Deutschland

2 Wirtschafts- und Sozialwissenschaftliches

Institut (WSI) Hans-Böckler-Stiftung,

Georg-Glock-Str. 18, 40474 Düsseldorf, Deutschland 


\title{
Work intensity and its determinants. An overview of the analytical potential of employment surveys
}

\begin{abstract}
High work intensity can be a health hazard for employees. In designing work to promote health, it is crucial to identify determinants of increased work intensity. This article deals with the question of what empirical database exists to describe the distribution and in particular the determinants of work intensity in Germany. For this purpose, six employment surveys were systematically analysed. A total of 662 items on the topic of "work intensity and its determinants" were identified and classified according to 11 main categories-six categories for work intensity and five for determinants-with more than 65 sub-categories. It turned out that there is already a broad database available to deal with the topic of work intensity and its determinants in Germany. Nevertheless, empirical gaps are also apparent. Future surveys should, among other aspects, collect information regarding performance control like costumer orientation as well business trips, target agreements at work, the degree of result orientation and self-responsibility as possible determinants of work intensity. The detailed survey of operational conditions, such as staff shortages, and business forms should also be given greater consideration in future surveys.

Practical Relevance: The present study shows which database is available in Germany to examine determinants of work intensity and thus also to identify the potential for an appropriate design of work intensity.
\end{abstract}

Keywords Work requirements $\cdot$ Workload $\cdot$ Work design $\cdot$ Health $\cdot$ Employment surveys

\section{Einleitung}

Die Arbeitswelt wird immer komplexer und befindet sich in einem stetigen Wandlungsprozess. Zunehmende Beschleunigung auf gesamtgesellschaftlicher Ebene, ständige Umstrukturierungen auf betrieblicher Ebene sowie ein sich immer schneller veränderndes Arbeitsumfeld erhöhen den Druck auf die Beschäftigten. Gleichzeitig verändern sich die Arbeitskontexte und sind beispielsweise durch eine direktere Kommunikation zwischen Kunden/-innen und Mitarbeiter/-innen gekennzeichnet (Rau 2017). Darüber hinaus werden die Produktions-, Service- und Kommunikationsprozesse kontinuierlich beschleunigt. Auch eine Zunahme der Komplexität der Aufgaben sowie steigende Lernanforderungen können beobachtet werden (Akzeleration; Rosa 2005). Folglich ist von einer zunehmenden quantitativen, aber auch qualitativen Intensivierung der Arbeit auszugehen und es mehren sich empirische Hinweise darauf (Ahlers 2016; Burke et al. 2009; Green 2006, 2001; Korunka und Kubicek 2017).

Es gibt vielfältige Hinweise darauf, dass eine hohe Arbeitsintensität eine gesundheitsgefährdende Belastung für Beschäftigte darstellen kann (Korunka und Kubicek 2017; Beermann et al. 2018; Seiler et al. 2013). Die negativen gesundheitlichen Folgen zu begrenzen ist eine zentrale Herausforderung für betriebliche und überbetriebliche Akteure. Jedoch fehlt derzeit - nicht zuletzt aufgrund begrifflicher Unschärfe - eine zusammenfassende Bewertung der vorliegenden empirischen Befunde. Ebenso fehlen umfassende Analysen zu den Determinanten von Arbeitsintensität. Der vorliegende Beitrag versucht erste Schritte, um diese Lücken zu schließen. So wird untersucht inwiefern sich verfügbare repräsentative Erwerbstätigenbefragungen eignen, um die Verbreitung und insbesondere die Determinanten von Arbeitsintensität in Deutschland zu beschreiben, um somit deren Analysepotential aber auch blinde Flecken aufzuzeigen.

\subsection{Arbeitsintensität}

Der bisherige Forschungsstand zeigt, dass derzeit keine allgemeingültige Beschreibung und ebenso keine gemeinsame übergreifende Theorie für Arbeitsintensität besteht, obwohl zu diesem Thema bereits zahlreiche Studien vorliegen (Dunkel und Kratzer 2016; Korunka et al. 2015; Soucek und Voss 2020). Folglich liegt auch kein einheitliches Konzept vor, wie Arbeitsintensität gemessen werden kann (Stab und Schulz-Dadaczynski 2017). In der Zusammenschau verschiedener qualitativer und quantitativer Studien zeigt sich, dass das Ausmaß der Arbeitsintensität von Quantität, Qualität und dem Tempo der zu leistenden Arbeit abhängig ist. Quantitative Anforderungen und Tempo umfassen die Menge, Geschwindigkeit und Zeit, in der eine bestimmte Arbeit zu verrichten ist. Qualitative Anforderungen umfassen die Komplexität, Schwere und Qualität der Arbeit (Stab und Schulz-Dadaczynski 2017). Spezifischer zeigen Rau und Göllner (Rau und Göllner 2018) auf, dass sich Arbeitsintensität anhand bestehender Studien weitestgehend als Arbeitsmenge, die pro Zeiteinheit zu erledigen ist, beschreiben lässt. Dabei bestimmt der für die Arbeitsausführung notwendige kognitive Aufwand (Hacker und Sachse 2014), ob mehr oder weniger Arbeit pro Zeiteinheit geleistet werden kann (Rau und Göllner 2018). Arbeitsintensität lässt sich somit auf drei zentrale Bedingungen zurückführen: Die Arbeitsmenge, die zur Verfügung stehende Zeit, in der die Menge bewältigt werden muss bzw. das Tempo bei 
der zu leistenden Arbeit und die Komplexität der Arbeitsaufgabe. Besteht eine Unvereinbarkeit dieser Bedingungen, erhöht sich die (subjektive) Arbeitsintensität (Dunkel und Kratzer 2016). Die sich aus der Unvereinbarkeit der Bedingungen ergebende psychische Belastung kann als negativ zu bewertende hohe Arbeitsintensität angesehen werden, da das Risiko besteht, dass die zur Bewältigung erforderliche Anstrengung und die damit einhergehende Beanspruchung zur Beeinträchtigung führt (Roe und Zijlstra 2000; Richter und Hacker 2012). Während eine hohe Arbeitsintensität zum Beispiel die Wahrnehmung von Zeit- oder Leistungsdruck beschreibt, ist Arbeitsintensivierung durch die Wahrnehmung einer Zunahme von Intensität charakterisiert (Korunka 2020).

Neben der Definition von Arbeitsintensität als Relation von Menge, Zeit/Tempo und Komplexität gibt es noch eine andere, objektivere Definition, die sich auf die Ausdehnung der Arbeitszeit bezieht. Genauer gesagt handelt es sich um die Verlängerung der Arbeitszeit, die anhand der Anzahl der geleisteten Arbeitsstunden gemessen werden kann und die sowohl eine Verlängerung der täglichen Arbeitszeit als auch das Arbeiten an freien Tagen umfasst. In der Konsequenz entfallen Erholungszeiten, insbesondere Pausen. Diese Form wird häufig als Extensivierung von Arbeit bezeichnet (Green 2001). Zu beachten ist, dass der Begriff Arbeitsintensität ebenfalls verwendet wird, um das gleichzeitige Auftreten eines erhöhten Arbeitseinsatzes innerhalb vorgegebener Zeiteinheiten und eine Verlängerung der Arbeitszeiten zu beschreiben (Korunka und Kubicek 2013).

\subsection{Determinanten von Arbeitsintensität}

Als Ursache einer hohen Arbeitsintensität werden aktuell eine Vielzahl von Faktoren diskutiert (für einen Überblick siehe Soucek und Voss 2020). Unter anderem wird der zunehmende Konkurrenz-, Kosten- und Leistungsdruck als Ursache gesehen, dem Unternehmen durch einen verstärkten globalen Wettbewerb und technologische Beschleunigung ausgesetzt sind und der oft an die Beschäftigten weitergeben wird. Im Rahmen dieser Logik ergreifen Unternehmen unter dem Druck des Marktes Kostensparmaßnahmen, etwa durch Abbau von Arbeitsplätzen. Das unveränderte Arbeitsvolumen wird auf die verbleibenden Beschäftigten umverteilt und von diesen (oft mit hohem Eigenengagement) bewältigt. Im Zuge dessen verändert sich vielerorts auch die Organisation von Arbeit mitsamt dem Arbeitsinhalt (Haipeter et al. 2017). Gleichzeitig entwickeln sich indirekte Steuerungsformen (Peters 2011), welche mit Arbeitsintensivierung in Verbindung gebracht werden können (Ahlers 2016). Dabei liegt die Durchschlagkraft dieser Wettbewerbs- und Globalisierungslogik auch in veränderten gesellschaftlichen und unternehmerischen Kulturen, Er- wartungen und Ideologien, die eine solche Ökonomisierung und Vermarktlichung stützen (Graefe 2010). Des Weiteren kann die Digitalisierung unter anderem durch die zunehmende Durchdringung der Arbeitswelt mit modernen Informations- und Kommunikationstechnologien (IKT), die andauernde Zunahme von Rechnerleistung oder den Einsatz von künstlicher Intelligenz bei der Arbeit zu einer Verdichtung von Arbeit führen (Carstensen 2015). Durch moderne IKT erweitern sich die Möglichkeiten des mobilen Arbeitens für Beschäftigte, was zu mehr Freiheiten und einer besseren Vereinbarkeit, gleichzeitig aber auch zur ständigen Erreichbarkeit und somit zu mehr Stress führen kann (Ahlers 2018). Außerdem nehmen die beruflichen Anforderungen mit dem Einsatz neuer IKT branchenübergreifend zu (Bosch et al. 2017). So zeigen Studien, dass die Berufe über die Zeit komplexer geworden sind (Spitz-Oener 2006; Helmrich et al. 2016). Passen beispielsweise die Anforderungen des Arbeitsplatzes nicht zu den Kompetenzen der Beschäftigten (Skill-Mismatch), kann der nachzuholende Qualifizierungsbedarf zu einer Ausweitung der Arbeitszeit führen, da das Lernen teilweise in der verfügbaren Arbeitszeit erfolgt (Rau und Göllner 2018, S. 168).

Ferner zeigen Studien, dass verschiedene individuelle und berufsbezogene Merkmale in Zusammenhang mit der Arbeitsintensität stehen (Institut DGB Gute Arbeit 2010; Eurofound 2015; Lohmann-Haislah 2012). Zum Beispiel verweisen diese Studien darauf, dass eine hohe Arbeitsintensität unter anderem häufiger bei Vollzeitbeschäftigten, höher Gebildeten und/oder Führungskräften auftritt. Stab und Schulz-Dadaczynski (Stab und Schulz-Dadaczynski 2017) zeigen zudem, dass im Berufsgruppenvergleich insbesondere Wissens- und Dienstleistungstätigkeiten von einer höheren Arbeitsintensität betroffen sind.

Insgesamt können zahlreiche Faktoren als Ursachen von Arbeitsintensität in Frage kommen. Diese Determinanten speisen sich aus gesellschaftlichen, betrieblichen, tätigkeitspezifischen und individuellen Merkmalen. Bei Betrachtung der bisherigen Forschungslage wird allerdings deutlich, dass sich die Mehrheit der quantitativen Studien zu Arbeitsintensität mit den (gesundheitlichen) Konsequenzen beschäftigen, die Ursachen von Arbeitsintensität hingegen werden häufig auf einer theoretischen Basis diskutiert. Hinsichtlich quantitativer empirischer Studien, welche die Determinanten von Arbeitsintensität beleuchten, besteht somit noch Forschungsbedarf. Insbesondere fehlen quantitative Studien zu den betrieblichen und tätigkeitsspezifischen Determinanten, wie zum Beispiel neue Steuerungsformen oder mobilem Arbeiten. Ausgehend von der bestehenden Forschungslücke, gehen wir der Frage nach, welche empirische Grundlage es aktuell in Deutschland gibt, um die Verbreitung und insbesondere die Determinanten von Arbeitsintensität in Deutschland zu beschreiben. 


\section{Methode}

Die in diesem Beitrag betrachtete Fragestellung fokussiert sich auf die Arbeitswelt. Somit sind insbesondere Datensätze von Relevanz, die explizit konzipiert wurden, um Informationen über den Arbeitskontext und die Arbeitsbedingungen von Erwerbstätigen zu erfassen. Dies trifft gerade auf Erwerbstätigenbefragungen zu, die der dezidierten Beschreibung des Arbeitslebens von Beschäftigten dienen.

Um das Analysepotential von Erwerbstätigenbefragungen für das Thema Arbeitsintensität und ihre Determinanten zu beleuchten, wurde eine systematische Sichtung von Erwerbstätigenbefragungen in Deutschland vorgenommen. Auswahlgrundlage für die zu sichtenden Datensätze bilden die in der Kurzexpertise „Berichterstattung über die Arbeitswelt in Deutschland: Datengrundlagen für Branchenbetrachtungen" (Bundesministerium für Arbeit und Soziales 2017) diskutierten Erwerbstätigenbefragungen. Im Rahmen der genannten Expertise wurden acht Erwerbstätigenbefragungen identifiziert, die Informationen zur Charakterisierung der Arbeitswelt beinhalten:

a) $\mathrm{BIBB} / \mathrm{BAuA}$-Erwerbstätigenbefragung

b) BAuA-Arbeitszeitbefragung

c) European Working Conditions Survey (EWCS)

d) DGB-Index Gute Arbeit (DBG-Index)

e) INQA - Gewünschte und erlebte Arbeitsqualität (INQAStudie)

f) European Skills and Jobs Survey (ESJS)

g) Lohnspiegel (WIF)

h) iga.Barometer

Die identifizierten Befragungen wurden in einem ersten Schritt einer gründlichen Überprüfung hinsichtlich der folgenden Kriterien unterzogen:

1. Abdecken des Themengebietes Arbeitsintensität und mögliche Determinanten

2. Repräsentativität mindestens für die abhängig beschäftigte Erwerbsbevölkerung

3. Bei europäischen Erhebungen das Vorliegen einer deutschen Teilstichprobe

4. Zugänglichkeit für interessierte Wissenschaftler/-innen

Der Einschluss einer Erhebung erfolgte nur, wenn alle vier Kriterien erfüllt waren. Anschließend wurde für die eingeschlossenen Datensätze ein mehrstufiges Kategoriensystem deduktiv und induktiv erstellt, um die Indikatoren für hohe Arbeitsintensität und deren Determinanten zu erfassen. Entlang dieses Kategoriensystems wurden in einem nächsten Schritt die Datensätze miteinander verglichen. Insgesamt wurden 11 Hauptkategorien gebildet: sechs Kategorien für Arbeitsintensität und fünf für Determinanten, die wiederum mehr als 65 Unterkategorien umfassen. Für die Sichtung der Datensätze wurde jeweils die aktu- ellste Erhebungswelle der einzelnen Befragungen, die zum Zeitpunkt der Analysen vorlagen, verwendet. Tab. 1 zeigt das Kategoriensystem mit seinen Haupt- und Unterkategorien. Ausgehend von den bereits beschriebenen drei zentralen Bedingungen von Arbeitsintensität wurden zunächst die Hauptkategorien Menge, Zeit/Tempo und Komplexität gebildet. Zudem wurde basierend auf dem bisherigen Forschungsstand ebenfalls vorab die Kategorie Extensivierung in das Kategoriensystem aufgenommen. Bei der Sichtung der Fragebögen wurde zu dem deutlich, dass es Items für Arbeitsintensität gibt, die gleichzeitig Informationen über Menge und Zeit/Tempo erfassen, somit wurde zudem die Hauptkategorie Menge/Zeit/Tempo gebildet, die zum Beispiel Fragen nach Termin- und Leistungsdruck beinhalten. Durch die Sichtung der Fragebögen zeigte sich ebenfalls, dass einige Datensätze Items beinhalten, die eine Veränderung der Arbeitsintensität über die Zeit abbilden. Diese wurden der Kategorie Arbeitsintensivierung zugeordnet.

Abschließend wurden die möglichen Determinanten der Arbeitsintensität, die in den gesichteten Fragebögen identifiziert werden konnten, in den Hauptkategorien Tätigkeitsmerkmale, betriebliche Rahmenbedingungen, Arbeitszeitmerkmale, Arbeitskontext und individuelle Merkmale (siehe Tab. 1) zusammengefasst.

Den entwickelten Haupt- und Unterkategorien wurden alle relevanten Items aus den Erwerbstätigenbefragungen zugeordnet. Ausschlaggebend für die Zuordnung war die thematische Passung. Da die gesichteten Items durchaus mehrere Themen in einem adressieren können, wurden manche Items zu unterschiedlichen Unterkategorien zugeordnet (z.B. Abfrage der tatsächlichen Arbeitszeit wurde zu Extensivierung und zu Arbeitszeitlänge zugeordnet).

\section{Ergebnisse}

Von den anfänglich identifizierten acht Erwerbstätigenbefragungen blieben unter Berücksichtigung der Einschlusskriterien sechs Befragungen übrig. Ausgeschlossen wurde das iga. Barometer, da es sich hier um keine freizugängliche Befragung handelt und ebenfalls wurde der Lohnspiegel (WIF) aufgrund der mangelnden Repräsentativität der Stichprobe nicht weiter einbezogen. Somit wurden in die folgenden tiefergehenden Betrachtungen die Datensätze der BIBB/BAuA-Erwerbstätigenbefragung 2018, der BAuA-Arbeitszeitbefragung 2017, des EWCS 2015, des DGB-Index 2019, der INQA Befragung 2013 und des ESJS 2014 einbezogen. Überwiegend handelt es sich bei den Befragungen um Querschnittsbefragungen bzw. wiederholte Querschnittsbefragungen. Nur zwei Studien sind als Längsschnittuntersuchungen angelegt (BAuA-Arbeitszeitbefragung und ESJS). Des Weiteren zeigt sich, dass die verschiedenen Datensätze neben dem Thema 
Tab. 1 Erfasste Kategorien und Unterkategorien für Arbeitsintensität und Determinanten in den Erhebungen

Table 1 Categories and subcategories for work intensity and determinants in the surveys

\begin{tabular}{|c|c|}
\hline Hauptkategorien & Unterkategorien \\
\hline \multicolumn{2}{|l|}{ Arbeitsintensität } \\
\hline Menge & z. B. Anforderungen durch die Arbeitsmenge gewachsen, Einfluss auf Arbeitsmenge \\
\hline Zeit/Tempo & z. B. sehr schnell arbeiten, hohes Arbeitstempo \\
\hline Menge/Zeit/Tempo & z. B. Termin-/Leistungsdruck \\
\hline Komplexität & z. B. Störungen/Unterbrechungen, Multitasking, komplexe Arbeitsaufgaben \\
\hline Arbeitsintensivierung & z. B. Zunahme Arbeitsdruck und Stress, mehr schaffen müssen als früher \\
\hline Extensivierung & z. B. lange Arbeitszeiten, Überstunden, Ausfall Pausen \\
\hline \multicolumn{2}{|l|}{ Determinanten } \\
\hline Tätigkeitsmerkmale & $\begin{array}{l}\text { Berufsklassifikation, Branchenklassifikation, Art der Tätigkeit, Privates/öffentliches Unternehmen, Geschlech- } \\
\text { teranteil Tätigkeit }\end{array}$ \\
\hline $\begin{array}{l}\text { Betriebliche Rahmenbedin- } \\
\text { gungen }\end{array}$ & $\begin{array}{l}\text { Personal-/Betriebsrat/Gewerkschaft vorhanden, Beauftragte/r für Arbeitsschutz und Gesundheit vorhanden, } \\
\text { Betriebsgröße, Aktiengesellschaft, Arbeitszeiterfassung/-modell/-regelung, wirtschaftliche Lage des Betriebs }\end{array}$ \\
\hline Arbeitszeitmerkmale & $\begin{array}{l}\text { Arbeitszeitlänge, Bereitschaftsdienst, Rufbereitschaft, Arbeit auf Abruf, atypische Arbeitszeiten, Nacht-/ } \\
\text { Schichtarbeit, Wochenendarbeit, Einfluss auf Arbeitszeit, Änderungen Arbeitszeiten }\end{array}$ \\
\hline Arbeitskontext & $\begin{array}{l}\text { (Ausmaß) Kundenorientierung, Personalengpässe, Dienstreisen, Ergebnisorientierung/Eigenverantwortung, } \\
\text { Zielvereinbarung, Leistungssteuerung/-kontrolle, Arbeiten mit Technik, gefühlsmäßige Belastung, Befristung, } \\
\text { Karrieremöglichkeiten, Qualifizierungsbedarf, Weiterbildungsmöglichkeiten/-teilnahme, Führungsqualität, } \\
\text { Gerechtigkeit, direkte Veränderungen am Arbeitsplatz/Information über Veränderungen, Umstrukturierung/ } \\
\text { Umorganisation (z. B. Personalabbau), Arbeitsplatzunsicherheit, sozialer Kontext (z. B. Projekt-/Teamarbeit, } \\
\text { Abhängigkeit von Kolleg/innen), Homeoffice/Telearbeit, Pendelweg, Mobiles Arbeiten/Arbeitsort, soziale Un- } \\
\text { terstützung/Einbindung, Unternehmenskultur/Betriebsklima, ständige Erreichbarkeit, Geschlecht Vorgesetzte/r, } \\
\text { Vereinbarkeit Familie und Beruf, Individuelle Gestaltungsmöglichkeiten }\end{array}$ \\
\hline Individuelle Merkmale & $\begin{array}{l}\text { Geschlecht, Alter, Schul-/Ausbildungsabschluss, Betriebszugehörigkeit, berufliche Stellung, Anforderungs-/ } \\
\text { Qualifikationsniveau, Einkommen, Führungsposition/Projektleitung/Führungsspanne, Karriereorientierung, } \\
\text { Nebentätigkeit, Mitglied Gewerkschaft, Familienstatus, soziale Unterstützung privates Umfeld }\end{array}$ \\
\hline
\end{tabular}

Arbeitsbedingungen unterschiedliche thematische Schwerpunktsetzungen haben. Während beispielsweise die BIBB/ BAuA-Erwerbstätigenbefragung die Kernthemen Arbeitsbedingungen, Arbeitsbelastung, Ausbildung/Weiterbildung sowie Gesundheit beinhaltet, fokussiert das ESJS auf Qualifikation, Qualifikationsdefizite und Arbeitsbedingungen. Hinweise auf weiterführende Informationen $\mathrm{zu}$ den verschiedenen Datensätzen sind in Tab. 2 zu finden sowie im Online Appendix.

Insgesamt wurden über alle sechs Datensätze hinweg 89 Items für Arbeitsintensität und 573 für Determinanten berücksichtigt und kategorisiert. Die meisten Items für Arbeitsintensität ließen sich in der BIBB/BAuA-Erwerbstätigenbefragung (26 Items) und die wenigsten im ESJS (7 Items) finden. Hinsichtlich der Determinanten war der EWCS (139 Items) am reichhaltigsten und das ESJS erhebt am wenigsten Determinanten für Arbeitsintensität (39 Items; siehe Tab. 2). ${ }^{1}$

Bei tiefergehender Betrachtung wird deutlich, dass Arbeitsintensität bzw. die Teilaspekte von Arbeitsintensität in den verschiedenen Studien in einem ganz unterschiedlichen Maße abgebildet werden. In allen eingeschlossenen

\footnotetext{
${ }^{1}$ Eine detaillierte Übersicht darüber, welche Items genau zu den einzelnen Kategorien zugeordnet wurden, befindet sich im Online-Appendix.
}

Datensätzen sind Aspekte erfasst, die sich der Kategorie Komplexität (z.B. Störungen/Unterbrechungen, komplexe Arbeitsaufgaben oder Probleme lösen) und Extensivierung (z. B. lange Arbeitszeiten, Verkürzung von Pausen oder Ruhezeiten) zuordnen lassen. Hinsichtlich Komplexität bietet die BIBB/BAuA-Erwerbstätigenbefragung mit 14 Items am meisten Auswertungsmöglichkeiten, bezüglich Extensivierung weist die BAuA-Arbeitszeitbefragung mit 12 Items am meisten Möglichkeiten auf. Mit Ausnahme des ESJS lassen sich in allen Befragungen Informationen zu Menge oder Zeit/Tempo bzw. Menge/Zeit/Tempo finden. Hier bietet das EWCS mit 3 Items in der Kategorie Zeit zum Beispiel verschiedene Möglichkeiten diesen Aspekt der Arbeitsintensität näher zu beleuchten (z. B. hohes Arbeitstempo, Zeit um Aufgaben zu erledigen und Termindruck). Items, die auf eine Arbeitsintensivierung schließen lassen, wie zum Beispiel Fragen nach der Zunahme der Arbeitsmenge oder von Arbeitsdruck werden in drei von sechs Befragungen erhoben.

Bezüglich der Determinanten zeigt sich ein sehr reichhaltiges Bild und in allen Befragungen konnten für alle Hauptkategorien (Tätigkeitsmerkmale, betriebliche Rahmenbedingungen, Arbeitszeitmerkmale, Arbeitskontext und individuelle Merkmale) Items gefunden werden. Zudem lassen sich für die Unterkategorien Branchenklassifikation, privates/öffentliches Unternehmen, Betriebsgröße, 


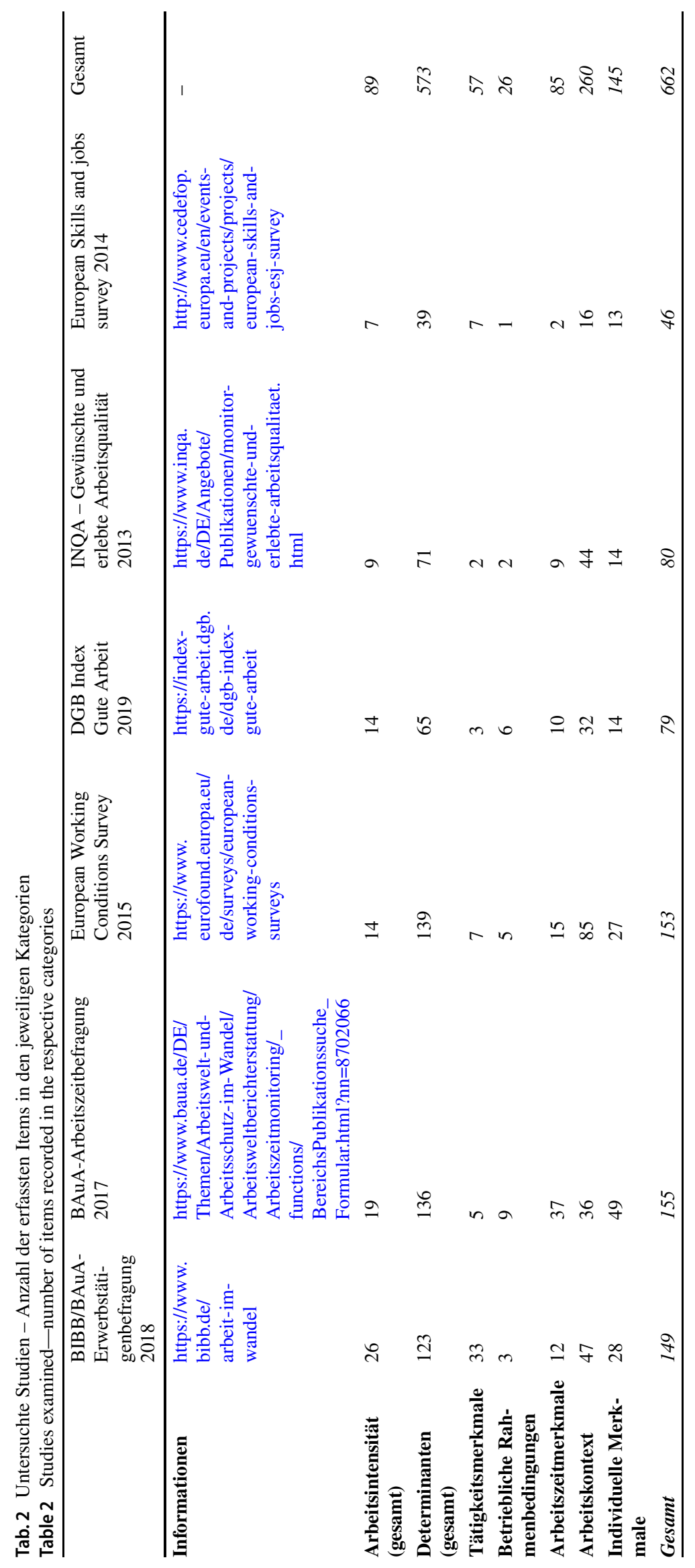


Arbeitszeitlänge, Befristung, Arbeitsplatzunsicherheit, Geschlecht, Alter, Schul-/Ausbildungsabschluss, berufliche Stellung, Einkommen und Führungsposition/Projektleitung/ Führungsspanne in allen Befragungen Variablen zuordnen. Allerdings ist an dieser Stelle darauf hinzuweisen, dass die Informationen zum Teil mit sehr unterschiedlichen Frageformulierungen erhoben worden sind und vergleichende Analysen über mehrere Befragungen somit nur eingeschränkt möglich sind (Hünefeld et al. 2020). Welche Determinanten für Arbeitsintensität in den jeweiligen Erwerbstätigenbefragungen abgebildet werden, hängt auch von den Schwerpunktsetzungen der Befragungen ab. So bietet sich die BIBB/BAuA-Erwerbstätigenbefragung insbesondere für die Betrachtung von Tätigkeitsmerkmalen (33 Items) als Determinanten an. Insbesondere die Art der Tätigkeit wird in dieser Befragung mit 24 Items umfänglich erhoben.

Erwartungsgemäß bietet die BAuA-Arbeitszeitbefragung am meisten Items zum Thema Arbeitszeit (39 Items). Ebenfalls eignet sich diese Befragung besonders zur Analyse der betrieblichen Rahmenbedingungen, insbesondere hinsichtlich Arbeitszeiterfassung, -modelle bzw. -kontrolle.

Für den Arbeitskontext weist der EWCS am meisten Items auf (85 Items). Dieser Datensatz beinhaltet insbesondere reichhaltige Informationen $\mathrm{zu}$ Gerechtigkeit bei der Arbeit (10 Items) sowie dem Betriebsklima und der Unternehmenskultur (15 Items). Darüber hinaus gibt es weitere Determinanten, die nur mit einzelnen Erwerbstätigenbefragungen beleuchtet werden können:

- BIBB/BAuA-Erwerbstätigenbefragung: Wirtschaftliche Lage des Betriebes (gefragt wurden nur Selbstständige)

- BAuA-Arbeitszeitbefragung: Soziale Unterstützung durch das private Umfeld

- EWCS: Geschlechteranteil in der beruflichen Tätigkeit, Vorhandensein eines/einer Beauftragten für Arbeitsschutz und Gesundheit, Ergebnisorientierung und Eigenverantwortung, Geschlecht Vorgesetzte/r

- DGB-Index: Leistungssteuerung/-kontrolle, Mitglied bei einer Gewerkschaft

Ferner gibt es einzelne Unterkategorien von möglichen Determinanten, die in keiner der Befragungen abgedeckt sind. So beinhalten die gesichteten Erwerbstätigenbefragungen keine Informationen darüber, ob es sich bei den Unternehmen um Aktiengesellschaften handelt und ob Personalengpässe vorliegen (Kratzer 2016). Allerdings bietet der DGB-Index von 2018 mit der Frage ,Wie häufig kommt es bei ihrer Arbeit mit (Kunden/Patienten, etc.) vor, dass der Arbeitsstress zunimmt, weil Sie wegen einer zu knappen Personalbemessung Ihre Arbeit nicht gut ausführen können?" die Möglichkeit sich mit dem Thema Personalengpässe in Teilen tiefergehend zu beschäftigten.

\section{Diskussion}

Die systematische Übersicht zeigt, dass die aktuellen und zugänglichen Erwerbstätigenbefragungen für Deutschland eine Vielzahl von Items liefern, um die Intensität bei der Arbeit und ihre möglichen Determinanten abzubilden. Mit den hier betrachteten Befragungen können alle Teilaspekte der Arbeitsintensität (Menge, Zeit/Tempo, Menge/Zeit/ Tempo, Komplexität, Arbeitsintensivierung und Extensivierung) beleuchtet werden, um die Verbreitung bzw. das Ausmaß von Arbeitsintensität in Deutschland abschätzen zu können. Gerade wenn man ein ganzheitliches Bild auf die Verbreitung der Arbeitsintensität haben möchte, bietet sich eine kombinierte Auswertung verschiedener Teilaspekte an. Auch dies können die vorliegenden Befragungen leisten und der DGB-Index sowie die BIBB/BAuA-Erwerbstätigenbefragung und die BAuA-Arbeitszeitbefragungen beinhalten jeweils Indikatoren für alle Teilaspekte. Ebenso können Zusammenhänge zwischen einzelnen Teilaspekten der Arbeitsintensität untersucht werden. Ein Beispiel hierfür ist die Untersuchung auf Basis der BIBB/BAuA-Erwerbstätigenbefragung, die sich mit dem Zusammenhang zwischen Termin-/Leistungsdruck, Multitasking und einem schnellen Arbeitstempo auf der einen Seite und langen Arbeitszeiten und dem Ausfall von Arbeitspausen auf der anderen Seite beschäftigt (Schulz-Dadaczynski et al. 2019).

Da die vorliegenden Erwerbstätigenbefragungen mehrheitlich als Querschnittsbefragungen angelegt sind, lässt sich mit ihnen vor allem die aktuelle Arbeitsintensität abbilden. Angaben $\mathrm{zu}$ einer Intensivierung von Arbeit sind hingegen schwieriger zu ermitteln. Von den hier betrachteten Erwerbstätigenbefragungen bietet aktuell nur die BAuA-Arbeitszeitbefragungen die Möglichkeit das Thema Arbeitsintensität im Längsschnitt zu betrachten. Somit besteht aktuell für Deutschland Nachholbedarf für Erwerbstätigenbefragungen, die als Längsschnitt angelegt sind. Positiv ist festzuhalten, dass anhand der wiederholten Querschnittsbefragungen Trendstudien zur Arbeitsintensivierung durchgeführt werden können und einige der Befragungen Indikatoren beinhalten, die retrospektiv die Zunahme der Intensität bei der Arbeit abfragen. Beispiele für bereits bestehende Analysen sind die Auswertung der Frage „Beinhaltet Ihre Arbeit Arbeiten unter Termindruck“ für die Wellen 1995, 2000, 2005, 2010 und 2015 des EWCS (Korunka 2020), die Betrachtung verschiedener Items, die für Arbeitsintensität stehen (z. B. Termin-/Leistungsdruck, sehr schnell Arbeiten oder Unterbrechungen/Störungen aus der BIBB/BAuA-Erwerbstätigenbefragung 2006, 2012 und 2018 [Hünefeld 2019]) sowie die Auswertung der Frage „Haben Sie den Eindruck, dass Sie in den letzten 12 Monaten mehr Arbeit in der gleichen Zeit als vorher schaffen müssen?" aus dem DGB-Index 2019 (Institut DGB-Gute Arbeit Index 2019). 
Hinsichtlich der Determinanten verdeutlicht die Übersicht, dass von Tätigkeitmerkmalen und betrieblichen Rahmenbedingungen (z.B. Branche oder die Betriebsgröße), über Arbeitszeitmerkmale (z.B. Länge oder Lage), bis hin zum Arbeitskontext (z. B. Ausmaß Kundenorientierung oder Führungsqualität) zahlreiche, für die Arbeitsintensität potenziell relevante Faktoren untersucht werden können. Unter anderem gibt es bereits Studien zum Zusammenhang zwischen (Teilaspekten von) Arbeitsintensität und der Einführung neuer Technologien (BIBB/BAuA-Erwerbstätigenbefragung 2018; Meyer et al. 2019), Arbeitszeiten außerhalb der Normalarbeitszeit (lange Arbeitszeiten, Nachtarbeit, Wochenendarbeit) (EWCS 2005-2015; Piasna 2018) sowie Zielvereinbarungen und Ausmaß der Kundenorientierung (Institut DGB-Gute Arbeit Index 2019).

Zum Teil lassen sich auch Analysen über mehrere Datensätze hinweg durchführen, um Ergebnisse aus verschiedenen Datensätze miteinander vergleichen zu können. So haben zum Beispiel Hünefeld und Kolleginnen (Hünefeld et al. 2020) die Verbreitung von hoher Arbeitsintensität in Deutschland anhand von annähernd vergleichbaren Items aus der BIBB/BAuA-Erwerbstätigenbefragung 2018, BAuA-Arbeitszeitbefragung 2015, dem EWCS 2015 und dem DGB-Index 2018 untersucht. Allerdings kommt diese Studie auch zu dem Ergebnis, dass trotz der Fülle von vorhandenen Items nur ein kleiner Anteil vergleichbarer Indikatoren für die Analysen gefunden werden konnten, da viele wichtige Determinanten nicht in allen Datensätzen erhoben wurden.

\section{Fazit}

Der vorliegende Beitrag verdeutlicht, dass in Deutschland bereits eine breite Datenbasis vorhanden ist, um sich mit dem Thema Arbeitsintensität und deren Determinanten weiterhin auseinanderzusetzen. Allerdings wurden im Verlauf dieser Analyse auch deutliche Datenlücken und Analysebarrieren erkennbar. Erstens, sind für die zukünftige Forschung mehr Längsschnitterhebungen wünschenswert, um sich dem Thema Arbeitsintensivierung tiefergehend widmen zu können. Zweitens, ist der Einsatz von gleichen bzw. ähnlichen Items in unterschiedlichen Befragungen zu erwägen, um eine bessere Vergleichbarkeit herzustellen und um zur Überprüfung der Robustheit von Ergebnissen gegenseitig validierende Abfragestrategien einsetzen zu können (Conrads et al. 2009).

Drittens, werden in der Gesamtschau dieser Datensätze aber auch inhaltliche Befragungslücken deutlich besonders in Bezug auf die Arbeitsorganisation und die Leistungserwartungen am Arbeitsplatz. Da eine gesundheitsförderliche Gestaltung der Arbeit insbesondere auf der betrieblichen Ebene stattfindet (Ulich und Wülser 2018) und die Arbeitsorganisation sowie die Steuerungsform zentrale Elemente bei der Gestaltung der Arbeitsintensität sind (Peters 2011; Kratzer 2020), sollten diese Elemente in Befragungen stärker Beachtung finden. Zukünftige Forschung sollte sich in quantitativen Erhebungen daher stärker auf die Arbeitsorganisation (z.B. ergebnisorientiertes Arbeiten, Zielvereinbarungen, Projektarbeit, agiles Arbeiten) und veränderte Leistungserwartungen (z. B. hohe Kundenorientierung, zusätzliche Dokumentationspflichten, regelmäßige Anpassung an neue Gegebenheiten) fokussieren. Zudem sind auch spezifische Komplexitätsaspekte der Tätigkeit (z.B. erforderliche Kooperations- und Koordinationsanforderungen oder Problemlösungskompetenzen) von Bedeutung. Des Weiteren sollte bei der Entwicklung neuer repräsentativer Erhebungen bzw. bei der Weiterentwicklung bestehender Befragungen eine (Re)Integration von Items zu den Themenfeldern Personalkapazitäten bzw. Personalengpässe sowie der Unternehmensform (z. B. Aktiengesellschaft) als mögliche Determinanten von Arbeitsintensität geprüft werden. Zusammenfassend kann diese vergleichende Arbeit mit dem Verweis auf systematische Datenlücken von hoher wissenschaftlicher Relevanz sein, die im Hinblick auf die Konzeptionierung zukünftiger Befragungswellen berücksichtigt werden kann.

Funding Open Access funding enabled and organized by Projekt DEAL.

Open Access Dieser Artikel wird unter der Creative Commons Namensnennung 4.0 International Lizenz veröffentlicht, welche die Nutzung, Vervielfältigung, Bearbeitung, Verbreitung und Wiedergabe in jeglichem Medium und Format erlaubt, sofern Sie den/die ursprünglichen Autor(en) und die Quelle ordnungsgemäß nennen, einen Link zur Creative Commons Lizenz beifügen und angeben, ob Änderungen vorgenommen wurden.

Die in diesem Artikel enthaltenen Bilder und sonstiges Drittmaterial unterliegen ebenfalls der genannten Creative Commons Lizenz, sofern sich aus der Abbildungslegende nichts anderes ergibt. Sofern das betreffende Material nicht unter der genannten Creative Commons Lizenz steht und die betreffende Handlung nicht nach gesetzlichen Vorschriften erlaubt ist, ist für die oben aufgeführten Weiterverwendungen des Materials die Einwilligung des jeweiligen Rechteinhabers einzuholen.

Weitere Details zur Lizenz entnehmen Sie bitte der Lizenzinformation auf http://creativecommons.org/licenses/by/4.0/deed.de.

\section{Literatur}

Ahlers E (2016) Arbeit und Gesundheit im betrieblichen Kontext. WSI Rep 33:1-15

Ahlers E (2018) Die Digitalisierung der Arbeit. Verbreitung und Einschätzung aus Sicht der Betriebsräte. WSI Rep 2018:1-22

Beermann B, Amlinger-Chaterjee M, Brenscheidt F, Gerstenberg S, Niehaus M, Wöhrmann AM (2018) Orts- und zeitflexibles Arbeiten: Gesundheitliche Chancen und Risiken. Bundesanstalt für Arbeitsschutz und Arbeitsmedizin, Dortmund

Bosch G, Bromberg T, Haipeter T, Schmitz J (2017) Industrie und Arbeit 4.0 Befunde zu Digitalisierung und Mitbestimmung im In- 
dustriesektor auf Grundlage des Projekts „Arbeit 2020“. In: IAQReport 2017, S 1-24

Bundesministerium für Arbeit und Soziales (2017) Berichterstattung über die Arbeitswelt in Deutschland: Datengrundlagen für Branchenbetrachtungen. Forschungsbericht 488. Bundesministerium für Arbeit und Soziales, Berlin

Burke RJ, Koyuncu M, Fiksenbaum L, Acar FT (2009) Work hours, work intensity, satisfactions and psychological well-being among Turkish manufacturing managers. Eur J Psychol 5:12-30

Carstensen T (2015) Neue Anforderungen und Belastungen durch digitale und mobile Technologien. WSI 68:187-193

Conrads R, Kistler E, Mußmann F (2009) Zum Stand der quantitativen Forschung über die Qualität der Arbeitsbedingungen in Deutschland. In: Bundesanstalt für Arbeitschutz und Arbeitsmedizin (Hrsg) Nutzerpotenziale von Beschäftigtenbefragungen. Repräsentative Beschäftigtenbefragungen als wichtige Informationsquelle zur Ermittlung der Arbeitsqualität - Workshop vom 20. Februar 2008 in Dortmund, S 11-28

DGB-Index Gute Arbeit (2010) Report 2010: Die Ergebnisse der Repräsentativerhebung. Schwerpunkte: Wirtschaftskrise und Arbeitsintensivierung. https://index-gute-arbeit.dgb.de/++co++b0 1ac12a-d764-11e3-b2c9-52540023ef1a. Zugegriffen: 8. Juni 2020

DGB-Index Gute Arbeit (2019) Report 2019. Arbeiten am Limit. Themenschwerpunkt Arbeitsintensität. https://index-gute-arbeit.dgb. de/veroeffentlichungen/jahresreports/++co++caa19028-151111ea-81ba-52540088cada. Zugegriffen: 8. Juni 2020

Dunkel W, Kratzer N (2016) Zeit- und Leistungsdruck bei Wissensund Interaktionsarbeit. Neue Steuerungsformen und subjektive Praxis. Nomos, Baden-Baden

Eurofound (2015) Sechste Europäische Erhebung über die Arbeitsbedingungen 2015. https://www.eurofound.europa.eu/de/data/ european-working-conditions-survey. Zugegriffen: 8. Juni 2020

Graefe S (2010) An den Grenzen der Verwertbarkeit. Erschöpfung im flexiblen Kapitalismus. In: Becker K, Gertenbach L, Laux H, Reitz T (Hrsg) Grenzverschiebungen des Kapitalismus. Umkämpfte Räume und Orte des Widerstands. Campus, Frankfurt a.M., S 229-252

Green F (2001) It's been a hard day's night: the concentration and intensification of work in late twentieth-century Britain. Br J Ind Relat 39:53-80

Green F (2006) Demanding work: the paradox of job quality in the affluent economy. Princeton University Press, Princeton

Hacker W, Sachse P (2014) Allgemeine Arbeitspsychologie. Psychische Regulation von Tätigkeiten. Hogrefe, Göttingen

Haipeter T, Bromberg B, Slomka C (2017) Angestellte als Machtquelle. Springer VS, Wiesbaden

Helmrich R, Tiemann M, Troltsch K et al (2016) Digitalisierung der Arbeitslandschaften. Keine Polarisierung der Arbeitswelt, aber beschleunigter Strukturwandel und Arbeitsplatzwechsel. BIBB Wissenschaftliche Diskussionspapiere, Bd. 180

Hünefeld L (2019) Zeitdruck und Co - Wird Arbeiten immer intensiver und belastender? BIBB/BAuA-Faktenblatt, Bd. 26. Bundesanstalt für Arbeitsschutz und Arbeitsmedizin, Dortmund

Hünefeld L, Meyer S-C, Ahlers E, Erol S (2020) Arbeitsintensität als Gegenstand empirischer Erhebungen. Das Potenzial repräsentativer Erwerbstätigenbefragungen für die Forschung. WSI 73:19-28

Korunka C (2020) Arbeitsintensivierung: Ursachen, Verläufe und Risikogruppen. WSI 73:11-18

Korunka C, Kubicek B (2013) Beschleunigung im Arbeitsleben. Neue Anforderungen und deren Folgen. In: Junghanns G, Morschhäuser M (Hrsg) Immer schneller, immer mehr. Psychische Belastung bei Wissens- und Dienstleistungsarbeit. Springer, Wiesbaden, S $17-41$

Korunka C, Kubicek B (2017) Job demands in a changing world of work. In: Korunka C, Kubicek B (Hrsg) Job demands in a changing world of work. Springer, Cham, S 1-5

Korunka C, Kubicek B, Paškvan M, Ulferts H (2015) Changes in work intensification and intensified learning: challenge or hindrance demands? Journal of Managerial Psych 30:786-800

Kratzer N (2016) Unternehmenskulturelle Aspekte des Umgangs mit Zeit- und Leistungsdruck. In: Badura B, Ducki A, Schröder H, Klose J (Hrsg) Fehlzeiten-Report 2016. Springer, Wiesbaden, S 21-31

Kratzer N (2020) Arbeitsintensität und Arbeitsintensivierung. WSI 73:3-10

Lohmann-Haislah A (2012) Stressreport Deutschland 2012. Bundesanstalt für Arbeitsschutz und Arbeitsmedizin, Berlin

Meyer S-C, Tisch A, Hünefeld L (2019) Arbeitsintensivierung und Handlungsspielraum in digitalisierten Arbeitswelten - Herausforderung für das Wohlbefinden von Beschäftigten? Industrielle Beziehungen. Z Arb Organisation Manag 2:207-231

Peters K (2011) Indirekte Steuerung und interessierte Selbstgefährdung. Eine 180-Grad-Wende bei der betrieblichen Gesundheitsförderung. In: Katzer N, Dunkel W, Becker K, Hinrichs S (Hrsg) Arbeit und Gesundheit im Konflikt: Analysen und Ansätze für ein partizipatives Gesundheitsmanagement. Nomos, Berlin, S $105-122$

Piasna A (2018) Scheduled to work hard: the relationship between non-standard working hours and work intensity among European workers (2005-2015). Hum Resour Manag J 28:167-181

Rau R (2017) Zum Stellenwert von Erholung in der Welt der Arbeit 4.0. In: Romahn R (Hrsg) Arbeitszeit gestalten. Wissenschaftliche Erkenntnisse für die Praxis. Metropolis, Marburg, S 61-77

Rau R, Göllner C (2018) Rahmenmodell der Arbeitsintensität als objektiv bestehende Anforderung. Arbeit 27:151-174

Richter P, Hacker W (2012) Belastung und Beanspruchung: Stress, Ermüdung und Burnout im Arbeitsleben. Asanger, Kröning

Roe RA, Zijlstra FRH (2000) Work pressure. Results of a conceptual and empirical analysis. In: Vartiainen M, Avallone F, Anderson N (Hrsg) Innovative theories, tools, and practices in work and organizational psychology. Hogrefe \& Huber, Ashland, S 29-45

Rosa H (2005) Beschleunigung: die Veränderung der Zeitstrukturen in der Moderne. Suhrkamp, Berlin

Schulz-Dadaczynski A, Wendsche J, Lohmann-Haislah A, Stab N (2019) Drivers of working longer: results from a large-scale and representative German employee survey. J Manag Organ. https:// doi.org/10.1017/jmo.2019.34:1-20

Seiler K, Beerheide E, Figgen M et al (2013) Arbeit, Leben und Erholung. Ergebnisse einer Repräsentativbefragung in NordrheinWestfalen. LIA.NRW, Düsseldorf

Soucek R, Voss AS (2020) Arbeitsverdichtung: Ursachen, Formen und Folgen. In: Arbeitsmedizin, Sozialmedizin, Umweltmedizin. 55: 543-546. https://www.asu-arbeitsmedizin.com/praxis/ arbeitsverdichtung-ursachen-formen-und-folgen. Zugegriffen: 14. Dez. 2020

Spitz-Oener A (2006) Technical change, job tasks, and rising educational demands: looking outside the wage structure. J Labor Econ 24:235-270

Stab N, Schulz-Dadaczynski A (2017) Arbeitsintensität: Ein Überblick zu Zusammenhängen mit Beanspruchungsfolgen und Gestaltungsempfehlungen. Z Arb Wiss 71:14-25

Ulich E, Wülser M (2018) Gesundheitsmanagement im Unternehmen. Arbeitspsychologische Perspektiven. Springer, Wiesbaden 